\title{
Soluções nutritivas para cultivo e produção de frutanos em plantas de Vernonia herbacea
}

\author{
Geraldo Rogério Faustini Cuzzuol(1), Maria Angela Machado Carvalho(2), Lilian Beatriz Penteado Zaidan(2) \\ e Pedro Roberto Furlani(3)
}

\begin{abstract}
(1)Universidade Federal do Espírito Santo, Setor de Botânica, Dep. de Ciências Biológicas/CCHN, CEP 29060-900 Vitória, ES. E-mail: cuzzuol@npd.ufes.br(2)Instituto de Botânica, Caixa Postal 4005, CEP 01061-970 São Paulo, SP. E-mail: lilianzaidan@uol.com.br, mam.carvalho@gmail.com ${ }^{(3)}$ Instituto Agronômico, Caixa Postal 28, CEP 13001-970 Campinas, SP. E-mail: pfurlani@iac.sp.gov.br
\end{abstract}

Resumo - O crescimento limitado de rizóforos de Vernonia herbacea (Asteraceae) em solução de Hoagland levou à necessidade de estabelecer uma solução nutritiva para o cultivo dessa planta, visando ao incremento da biomassa de seus rizóforos ricos em frutanos. Essa solução (denominada Vernonia), constituída de

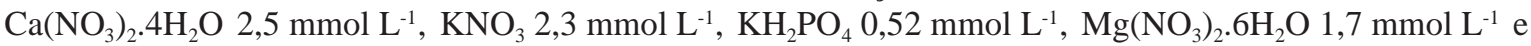
$\mathrm{Na}_{2} \mathrm{SO}_{4} 1,3 \mathrm{mmol} \mathrm{L}^{-1}$, foi comparada com a de Hoagland nas forças iônicas de 50\%, $100 \%$ e 200\%. Foram realizadas duas avaliações para análise de crescimento e conteúdo de frutanos. As plantas não sobreviveram até os dois meses na solução de Hoagland 200\%. A solução Vernonia diluída duas vezes (50\%) foi a mais eficiente para o incremento de massa seca dos rizóforos e produção de frutanos por planta. Maior crescimento da parte aérea foi verificado nas soluções de Hoagland e Vernonia 100\%. Em comparação com a solução de Hoagland, a solução Vernonia é mais pobre em macronutrientes, confirmando a hipótese de que plantas adaptadas a solos oligotróficos são menos exigentes em nutrientes minerais.

Termos para indexação: nutrição mineral, Asteraceae, cerrado.

\section{Nutrient solutions for plant growth and fructan production in Vernonia herbacea}

\begin{abstract}
The limited growth of rhizophores of Vernonia herbacea in Hoagland solution demanded the definition of a nutrient solution for plants of $V$. herbacea, aiming at the increase of the rhizophore biomass and fructan production. This solution, named Vernonia, is comprised of $\mathrm{Ca}\left(\mathrm{NO}_{3}\right)_{2} \cdot 4 \mathrm{H}_{2} \mathrm{O} 2.5 \mathrm{mmol} \mathrm{L}{ }^{-1}, \mathrm{KNO}_{3} 2.3 \mathrm{mmol} \mathrm{L}^{-1}$, $\mathrm{KH}_{2} \mathrm{PO}_{4} 0.52 \mathrm{mmol} \mathrm{L}^{-1}, \mathrm{Mg}\left(\mathrm{NO}_{3}\right)_{2} \cdot 6 \mathrm{H}_{2} \mathrm{O} 1.7 \mathrm{mmol} \mathrm{L}-1$ and $\mathrm{Na}_{2} \mathrm{SO}_{4} 1.3 \mathrm{mmol} \mathrm{L}{ }^{-1}$. Its effect on plants was compared to that of Hoagland solution, both with different ionic strengths, 50\%, 100\% and 200\%. The effect of the solutions on plant growth and fructan content was evaluated twice in a six-month period. Plants did not survive up to two months, when cultivated in 200\% Hoagland solution. The 50\% Vernonia solution was the most effective for rhizophore biomass increase and fructan production per plant. Growth of aerial organs was promoted in $100 \%$ Hoagland and Vernonia solutions. Compared to Hoagland, Vernonia solution contains less macronutrients, which confirms the hypothesis that plants adapted to the oligotrophic soils of the cerrado, as $V$. herbacea, demand less mineral nutrients to achieve full growth.
\end{abstract}

Index terms: mineral nutrition, Asteraceae, cerrado.

\section{Introdução}

Soluções nutritivas vêm sendo amplamente usadas em estudos de fisiologia vegetal, especialmente nos relacionados aos mecanismos que coordenam o crescimento das plantas (Parker \& Novell, 1999).

Diversas soluções nutritivas já foram propostas em pesquisas de nutrição mineral de plantas, havendo, em alguns casos, diferenças marcantes entre elas, no que se refere aos macronutrientes. Grande parte das for- mulações existentes é modificação da solução original de Hoagland, desenvolvida a partir das exigências nutricionais do tomateiro. Esta última é a mais usada na investigação de problemas nutricionais de plantas e configura-se como a base para formulação de várias soluções nutritivas comerciais (Furlani et al., 1999).

Segundo Hoagland \& Arnon (1938), não há uma composição nutritiva melhor do que outra, pois as plantas possuem grande capacidade de adaptação às diferentes condições nutricionais. Nos primeiros estudos, a com- 
posição química das soluções nutritivas simulava a constituição da solução do solo; atualmente, a composição de uma solução nutritiva baseia-se, preferencialmente, na composição química das folhas por serem os órgãos que melhor refletem o estado nutricional da planta (Parker \& Norvell, 1999). Segundo Martinez (1997), as maiores diferenças entre esses dois tipos de solução reside nos teores de N, P e K, com concentrações mais elevadas nas que se baseiam na composição química foliar.

Em geral, plantas de ambientes oligotróficos como a restinga, o manguezal e o cerrado acumulam menos nutrientes em relação a cultivares agrícolas, refletindo, desse modo, o estado nutricional do solo em que se encontram.

Com relação ao cerrado, vários estudos descreveram o efeito da adição de solução nutritiva de Hoagland sobre o crescimento inicial de plantas arbóreas, não tendo havido, em geral, o favorecimento do crescimento. Solução de Hoagland em 100\% de sua força iônica levou à abscisão ou ao amarelecimento precoce das folhas em plantas de Dalbergia miscolobium, tendo ocorrido melhor resposta de crescimento da parte aérea com solução de Hoagland diluída duas vezes (Sassaki \& Felippe, 1998). Em Qualea grandiflora, a solução de Hoagland, diluída dez vezes, provocou maior crescimento em todos os órgãos e na razão raiz:parte aérea (Paulilo \& Felippe, 1995).

Estudos realizados com herbáceas de cerrado apontam para um efeito promotor no crescimento da parte aérea, quando a solução nutritiva é aplicada. $\mathrm{O}$ crescimento de Bidens gardneri (Asteraceae), em resposta à adição da solução de Hoagland no solo, foi diretamente proporcional à força iônica da solução (Klein et al., 1996). Embora em Vernonia herbacea, outra Asteraceae nativa de cerrado, a massa seca da parte aérea tenha sido maior em plantas que receberam solução de Hoagland completa, a massa seca de seus rizóforos não aumentou em relação às plantas controle (Teixeira et al., 1997).

Rizóforos de $V$. herbacea são órgãos subterrâneos de origem caulinar, ricos em frutanos (Carvalho \& Dietrich, 1993). Frutanos são polímeros de frutose associados a mecanismos de resistência à baixa temperatura e dessecação (Hendry \& Wallace, 1993) e, também, à deficiência nutricional (McGrath et al., 1997). Por suas propriedades nutricionais e medicinais, o uso de frutanos tem sido recomendado nas dietas de baixa caloria, na prevenção de doenças intestinais e no tra- tamento da osteoporose (Tomomatsu, 1994). Um levantamento florístico realizado em área de cerrado em Moji Guaçu, SP, mostrou que 60\% das Asteraceae acumulam frutanos do tipo inulina em seus órgãos subterrâneos de reserva (Tertuliano \& Figueiredo-Ribeiro, 1993).

O crescimento insatisfatório de rizóforos de $V$. herbacea, em solução de Hoagland (Teixeira et al. 1997), e o interesse em se obter aumento da biomassa dos rizóforos e da produção de frutanos justificam a pesquisa nesta área.

O objetivo deste trabalho foi avaliar o efeito de uma solução nutritiva elaborada especificamente para $V$. herbacea, a fim de aumentar a biomassa de seus rizóforos e a produtividade em frutanos.

\section{Material e Métodos}

Plantas de Vernonia herbacea (Vell.) Rusby (Asteraceae) foram obtidas por propagação vegetativa a partir de fragmentos de rizóforos de plantas coletadas na Reserva Biológica e Estação Experimental de Moji

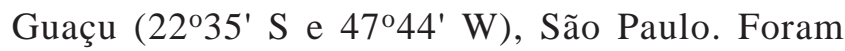
selecionadas plantas de tamanho uniforme (aproximadamente $5 \mathrm{~cm}$ de altura), aos 50 dias após a brotação dos rizóforos cultivados em bandejas com areia lavada. As plantas foram transferidas para vasos de plástico (1 L), contendo areia lavada como substrato, e submetidas às condições de luz, temperatura e fotoperíodo natural em casa de vegetação, com médias de temperatura mínima e máxima anual de $18^{\circ} \mathrm{C}$ e $31^{\circ} \mathrm{C}$, respectivamente. O experimento iniciou-se em outubro de 2000 e encerrou-se em março de 2001.

Conforme a análise química de plantas de $V$. herbacea [( $\mathrm{g} \mathrm{kg}^{-1}, \mathrm{~N}(21,7), \mathrm{P}(2,4), \mathrm{K}(15,0), \mathrm{Ca}(13,8), \mathrm{Mg}(6,3)$, $\mathrm{S}(5,7)$ e $\mathrm{mg} \mathrm{kg}^{-1}, \mathrm{~B}$ (31), Cu (18), Fe (802), Mn (88), Zn (44)], realizada pelo Laboratório do Centro de Pesquisa e Desenvolvimento de Solos e Recursos Ambientais, do Instituto Agronômico, em Campinas, foi elaborada uma solução nutritiva específica para essa espécie, de acordo com a sua constituição química foliar, na qual os valores expressos em mg L ${ }^{-1}$ são: $\mathrm{N}^{-N_{3}}{ }^{-}$, 150; P, 16; K, 102; Ca, 87; Mg, 40; e S, 43. Para essa solução, denominada Vernonia, usaram-se os seguintes sais: $\mathrm{Ca}\left(\mathrm{NO}_{3}\right)_{2} \cdot 4 \mathrm{H}_{2} \mathrm{O} 2,5 \mathrm{mmol} \mathrm{L}^{-1}, \mathrm{KNO}_{3} 2,3 \mathrm{mmol} \mathrm{L}^{-1}$, $\mathrm{KH}_{2} \mathrm{PO}_{4} 0,52 \mathrm{mmol} \mathrm{L}^{-1}, \mathrm{Mg}\left(\mathrm{NO}_{3}\right)_{2} \cdot 6 \mathrm{H}_{2} \mathrm{O} 1,7 \mathrm{mmol} \mathrm{L}^{-1} \mathrm{e}$ $\mathrm{Na}_{2} \mathrm{SO}_{4} 1,3 \mathrm{mmol} \mathrm{L}^{-1}$. Os sais de micronutrientes foram acrescentados de acordo com a solução de Hoagland (Hoagland \& Arnon, 1938). 
O efeito dessa solução no crescimento de $V$. herbacea e no conteúdo de frutanos foi comparado com o da solução de Hoagland, utilizando-se 50\%, 100\% e $200 \%$ da força iônica de ambas as soluções. Para maior controle da qualidade da água e das concentrações salinas da força iônica das soluções nutritivas, foi medida a condutividade elétrica de cada solução, determinada em mS cm-1 no condutivímetro Digimed CD-20, tendo-se obtido os seguintes valores: $50 \%$ Hoagland: 1,93; 50\% Vernonia: 0,77; 100\% Hoagland: 3,46; 100\% Vernonia: 1,28; 200\% Hoagland: 5,68 e 200\% Vernonia: 2,30. O pH das soluções foi ajustado para 5,5-6,0 com $\mathrm{KOH} 1 \mathrm{~N}$.

Cada planta recebeu, semanalmente, $50 \mathrm{~mL}$ da solução e, no dia anterior à aplicação dos tratamentos, as plantas foram regadas com água destilada, para evitar a salinização do substrato e dessecamento das plantas.

Nas análises de crescimento, foram utilizadas seis plantas por tratamento, para medidas de altura do maior ramo, massa de matéria seca dos órgãos aéreos e subterrâneos, número de folhas e área foliar total. A massa seca foi determinada após a secagem do material em estufa, a $60^{\circ} \mathrm{C}$, até obtenção de massa constante. A área foliar foi calculada pelo programa "Leaf Area \& Analysis” da Skye Instruments Ltd. (1993). As análises foram feitas após três e seis meses de cultivo. A partir desses dados, foram calculadas a taxa média de crescimento relativo $(\mathrm{TCR})=\left(\mathrm{LnM}_{2}-\mathrm{LnM}_{1}\right) /\left(\mathrm{t}_{2}-\mathrm{t}_{1}\right)$ e a taxa média de assimilação líquida (Hunt, 1982) $(T A L)=\left[\left(\operatorname{LnA}_{2}-\operatorname{Ln} A_{1}\right) /\left(A_{2}-A_{1}\right)\right] \times\left[\left(M_{2}-M_{1}\right) /\left(t_{2}-t_{1}\right)\right]$, em que $\mathrm{M}_{2}$ é a massa de matéria seca atual, $\mathrm{M}_{1}$ é a massa de matéria seca inicial, $A_{2}$ é a área foliar atual, $A_{1}$ é a área foliar inicial, $t_{2}-t_{1}$ é o intervalo de tempo entre duas coletas.

Os carboidratos solúveis foram extraídos segundo o método descrito por Carvalho et al. (1998), a partir de seis amostras (1,0 g de matéria fresca de rizóforos de cada planta). Os rizóforos foram fragmentados e fervidos em etanol $80 \%$ por 3 min, para desnaturação enzimática. Em seguida, foram macerados em almofariz, aquecidos em banho-maria a $80^{\circ} \mathrm{C}$ por $15 \mathrm{~min}$, e os homogeneizados foram centrifugados a $1.000 \mathrm{~g}$ por $10 \mathrm{~min}$, à temperatura ambiente. O resíduo foi re-extraído mais duas vezes, como descrito anteriormente, e então submetido duas vezes à extração aquosa por 30 min, a $60^{\circ} \mathrm{C}$, e filtrado a vácuo. Os sobrenadantes da fração etanólica e os filtrados aquosos foram reunidos, constituindo o extrato de frutanos totais e concentrados em rotoevaporador, a $40^{\circ} \mathrm{C}$. O conteúdo de frutose livre e combinada presente nos extratos foi estimado pela reação de antrona, modificada para cetoses (Jermyn, 1956), usando-se frutose (Sigma) como padrão.

O delineamento experimental foi inteiramente casualizado, com seis repetições. Os efeitos dos tratamentos foram submetidos à análise de variância e as médias comparadas pelo teste de Tukey a 5\%, utilizando-se o programa Sanest (Zonta et al., 1984).

\section{Resultados e Discussão}

Em relação a cultivares agrícolas (Raij et al., 1997), o teor de N $(21,7)$ e P $(2,4)$ foliar de V. herbacea foi inferior, mas superior ao encontrado em arbóreas de manguezal (Cuzzuol \& Campos, 2001) e de restinga (Moraes \& Domingos, 1997). Isto confirma informações anteriores de que o status nutricional das folhas reflete as condições nutricionais do ambiente onde a planta se desenvolve (Marschner, 1995).

A concentração de macronutrientes na solução Vernonia é inferior à de Hoagland, pois apresenta aproximadamente metade dos valores de $\mathrm{P}, \mathrm{K}$, Ca e S (Tabela 1). Essa informação é confirmada pelos valores de condutividade elétrica, sempre maiores na solução de Hoagland. As soluções de Hoagland e Vernonia apresentam concentrações de elementos químicos muito superiores às encontradas na solução de um solo fértil (Tabela 1). Maiores diferenças foram observadas no teor de $\mathrm{P}$, cujo valor foi cerca de 125 e 250 vezes maior na solução Vernonia e Hoagland, respectivamente.

Tabela 1. Concentrações de macronutrientes e micronutrientes nas soluções nutritivas de Hoagland (100\%) e Vernonia (100\%), e em solo fértil(1).

\begin{tabular}{|c|c|c|c|c|c|c|c|c|c|c|c|}
\hline \multirow[t]{2}{*}{ Soluções } & \multicolumn{6}{|c|}{ Macronutrientes $\left(\mathrm{mmol} \mathrm{L}^{-1}\right)$} & \multicolumn{5}{|c|}{ Micronutrientes $\left(\mu \mathrm{mol} \mathrm{L}{ }^{-1}\right)$} \\
\hline & $\mathrm{N}-\mathrm{NO}_{3}^{-}$ & $\mathrm{P}-\mathrm{H}_{2} \mathrm{PO}_{4}^{-}$ & $\mathrm{K}^{+}$ & $\mathrm{Ca}^{2+}$ & $\mathrm{Mg}^{2+}$ & $\mathrm{SO}_{4}{ }^{2-}$ & B & $\mathrm{Cu}$ & $\mathrm{Fe}$ & $\mathrm{Mn}$ & $\mathrm{Zn}$ \\
\hline Vernonia & 10,7 & 0,500 & 2,60 & 2,20 & 1,70 & 1,30 & 46,0 & 0,03 & 90,0 & 12,6 & 1,3 \\
\hline Hoagland & 15,0 & 1,000 & 6,00 & 5,00 & 2,00 & 2,00 & 46,0 & 0,03 & 90,0 & 12,6 & 1,3 \\
\hline Solo fértil & 0,5 & 0,004 & 0,09 & 1,13 & 0,26 & 0,13 & 10,0 & 0,30 & 5,0 & 5,0 & 0,4 \\
\hline
\end{tabular}

(1)Furlani et al. (1999). 
O efeito das soluções nutritivas nas medidas foliares de $V$. herbacea é apresentado na Figura 1. Diferenças significativas só foram manifestadas aos 180 dias de
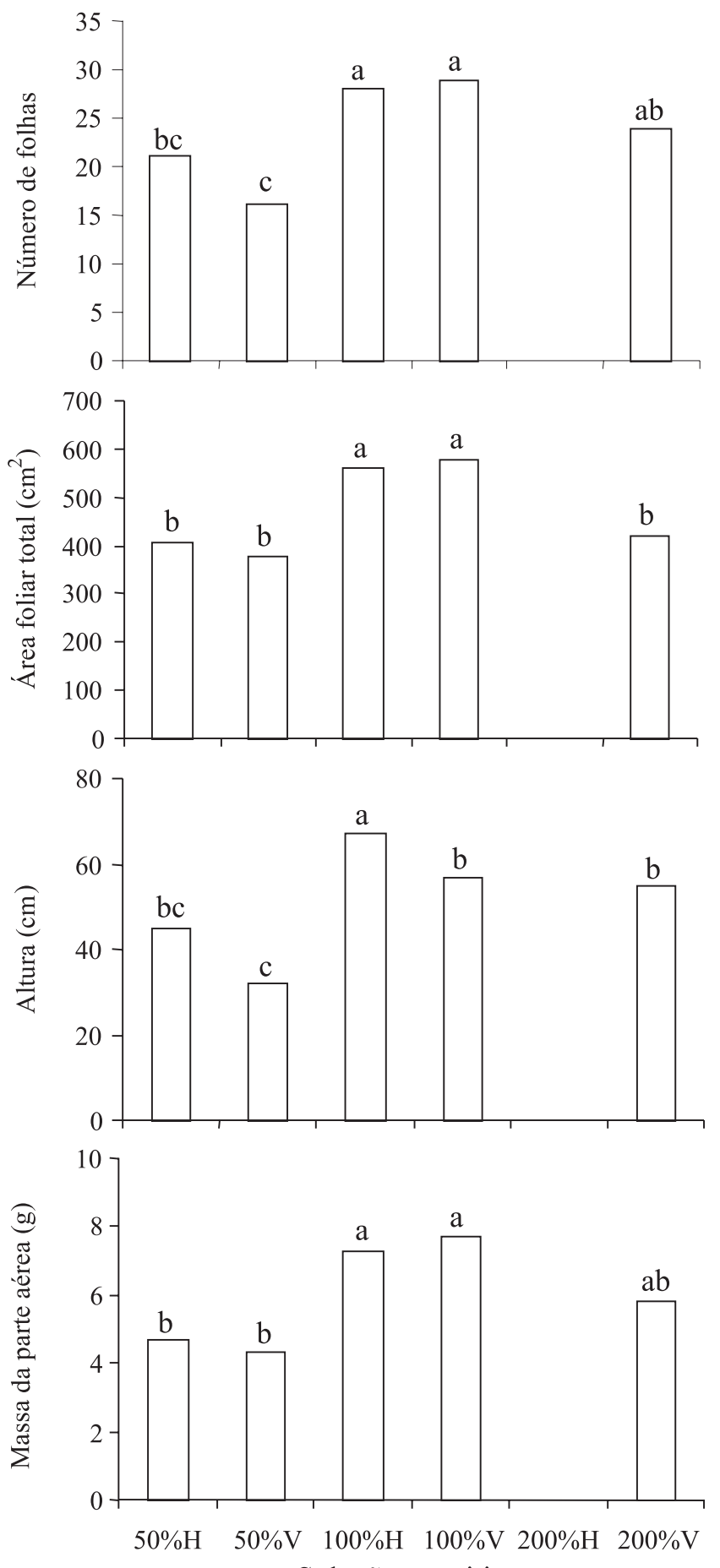

Soluções nutritivas cultivo, quando as soluções de Hoagland e Vernonia, com $100 \%$ de força iônica, levaram ao aumento do número de folhas e da área foliar total. No entanto, maior
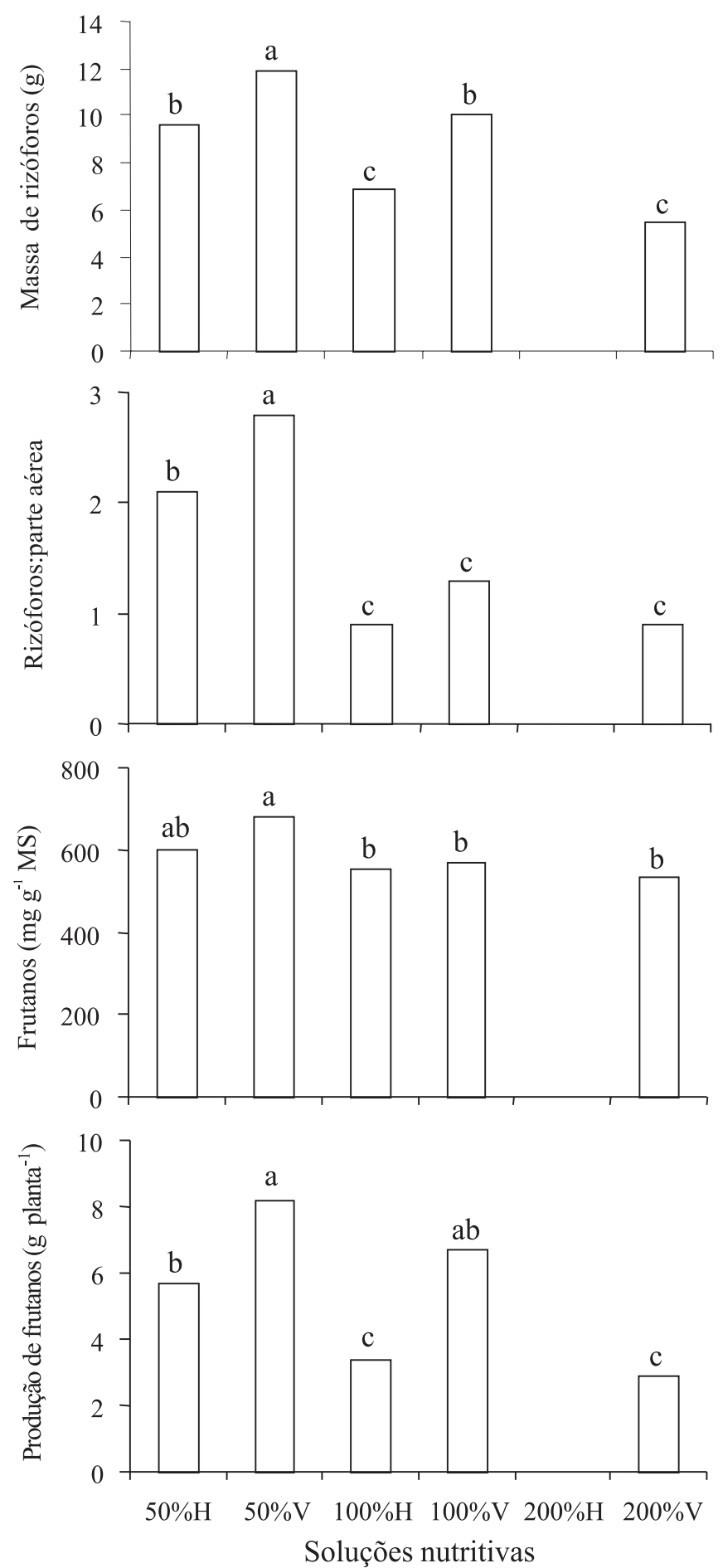

Figura 1. Efeito de soluções nutritivas no crescimento de folhas e de caule, na massa de matéria seca e na razão rizóforos:parte aérea, na concentração e na produção de frutanos em rizóforos de plantas de $V$. herbacea, após 180 dias de cultivo. Porcentagem da força iônica das soluções nutritivas de Hoagland (\%H) e Vernonia (\%V). Letras diferentes indicam diferenças significativas a $5 \%$ de probabilidade pelo teste de Tukey. 
área foliar unitária foi observada na solução Vernonia com $50 \%$ de força iônica, que chegou a apresentar 27,7\% de aumento em relação às soluções mais ricas em nutrientes. Efeito similar também foi constatado por Gomes \& Shepherd (2000) em plantas de Sinningia allagophylla (Gesneriaceae), cultivadas in vitro sob restrição nutricional, sugerindo um eficiente mecanismo fisiológico capaz de maximizar a interceptação luminosa, para atender à demanda do limitado crescimento da parte aérea nessas condições. Essa resposta é indicativa de maior eficiência na utilização dos nutrientes em $V$. herbacea sob restrição mineral, uma vez que um dos efeitos do nitrogênio é o de promover a expansão foliar (Marschner, 1995). Uma outra forma de V. herbacea contornar a deficiência mineral está na redução da emergência de novas folhas, o que refletiu na menor área foliar total. Na análise dos efeitos das soluções nutritivas na altura (Figura 1), nota-se melhor desempenho das plantas na condição em que receberam soluções mais ricas, principalmente na de Hoagland, com 100\% de força iônica.

Semelhantemente aos valores de crescimento foliar e caulinar, diferenças significativas quanto à produção de matéria seca também ocorreram somente aos 180 dias (Figura 1). Novamente, as soluções de Hoagland e Vernonia mais ricas em nutrientes elevaram a produção de massa seca da parte aérea, não tendo havido diferenças significativas entre elas. Por sua vez, soluções mais pobres em nutrientes estimularam maior partição de biomassa para os rizóforos, em detrimento dos órgãos aéreos, o que refletiu na maior razão rizóforos:parte aérea (Figura 1), especialmente a solução Vernonia com $50 \%$ de força iônica, em que as plantas chegaram a produzir 12 g de massa seca de rizóforos. Resultado similar foi observado no crescimento inicial da arbórea D. miscolobium, em que a razão raiz:parte aérea aumentou com a diminuição da força iônica da solução nutritiva de Hoagland (Sassaki \& Felippe, 1998), o que confirma Lambers et al. (1998) na constatação de que essa razão é maior sob deficiência nutricional.
Os resultados de crescimento se refletiram na TAL, e muito pouco na TCR (Tabela 2). Valores mais elevados de TCR, quanto ao caule e raiz, foram obtidos em soluções de Hoagland e Vernonia 100\%, enquanto no que diz respeito à folha, rizóforos e planta inteira, nenhuma diferença foi observada entre os diferentes tratamentos. Os valores de TCR da planta inteira ficaram ao redor de $0,0230 \mathrm{~g} \mathrm{~g}^{-1} \mathrm{~d}^{-1}$. Quanto à TAL, o valor mais elevado $\left(0,0074 \mathrm{~g} \mathrm{~cm}^{-2} \mathrm{~d}^{-1}\right)$ foi encontrado na solução Vernonia diluída à metade de sua força iônica. De acordo com Lambers et al. (1998), a TCR reflete a eficiência da planta em produzir matéria seca (TAL) e área assimilatória (RAF). Apesar de os valores mais baixos da TAL terem sido verificados nas soluções mais ricas em nutrientes, estes não influenciaram a TCR, pois nenhuma diferença foi observada nessa taxa entre as soluções testadas (Tabela 2). Isso sugere que a condição nutricional do solo parece não afetar a velocidade de crescimento em $V$. herbacea e que, nessa espécie, a TAL seja o componente mais sensível às variações ambientais.

Segundo Grime \& Hunt (1975), valores de TCR inferiores a $0,150 \mathrm{~g} \mathrm{~g}^{-1} \mathrm{~d}^{-1}$ são considerados baixos e as taxas mais elevadas, da ordem de $0,300 \mathrm{~g} \mathrm{~g}^{-1} \mathrm{~d}^{-1}$, têm sido encontradas em herbáceas. Desse modo, o baixo potencial de TCR em V. herbacea encontrado sustenta a hipótese de que espécies nativas, adaptadas a solos pobres em nutrientes, mostram baixa taxa de crescimento, mesmo quando a disponibilidade de nutrientes se torna maior (Marschner, 1995; Lambers et al., 1998).

Se, por um lado, soluções mais ricas em nutrientes promoveram o crescimento da parte aérea, o conteúdo de frutanos foi menor (Figura 1), o que confirma os resultados apresentados por Teixeira et al. (1997) em plantas de $V$. herbacea cultivadas em solução completa de Hoagland. Esses mesmos autores argumentaram que o crescimento dos órgãos aéreos, estimulados pela maior disponibilidade de nutrientes, resultaria em baixa translocação de assimilados para os rizóforos e, conseqüentemente, baixa síntese de frutanos. Cuzzuol et al.

Tabela 2. Taxa de crescimento relativo (TCR) e taxa assimilatória líquida (TAL) em plantas de Vernonia herbacea, cultivadas em soluções nutritivas ${ }^{(1)}$.

\begin{tabular}{|c|c|c|c|c|c|c|}
\hline \multirow[t]{2}{*}{ Tratamento } & \multicolumn{5}{|c|}{ TCR $\left(g^{-1} d^{-1}\right)$} & \multirow[t]{2}{*}{ TAL $\left(\mathrm{g} \mathrm{cm}^{-2} \mathrm{~d}^{-1}\right)$} \\
\hline & Folha & Caule & Raiz & Rizóforos & Planta & \\
\hline $50 \%$ Hoagland & $0,015 \mathrm{a}$ & $0,019 \mathrm{ab}$ & $0,013 b$ & $0,019 \mathrm{a}$ & $0,023 a$ & $0,0068 \mathrm{a}$ \\
\hline $50 \%$ Vernonia & $0,015 \mathrm{a}$ & $0,017 \mathrm{~b}$ & $0,015 \mathrm{ab}$ & $0,020 \mathrm{a}$ & $0,024 \mathrm{a}$ & $0,0074 \mathrm{a}$ \\
\hline $100 \%$ Hoagland & $0,017 \mathrm{a}$ & $0,024 \mathrm{a}$ & $0,022 \mathrm{a}$ & $0,017 \mathrm{a}$ & $0,023 \mathrm{a}$ & $0,0052 b$ \\
\hline $100 \%$ Vernonia & $0,018 \mathrm{a}$ & $0,023 \mathrm{a}$ & $0,022 \mathrm{a}$ & $0,019 \mathrm{a}$ & $0,024 \mathrm{a}$ & $0,0068 \mathrm{a}$ \\
\hline $200 \%$ Vernonia & $0,016 \mathrm{a}$ & $0,022 \mathrm{a}$ & $0,011 \mathrm{~b}$ & $0,016 \mathrm{a}$ & $0,021 \mathrm{a}$ & $0,0052 b$ \\
\hline
\end{tabular}

(1)Letras diferentes indicam diferenças significativas a 5\% de probabilidade pelo teste de Tukey. 
(2003) demonstraram que concentrações de N que promovem o crescimento não favorecem o acúmulo de frutanos. O maior acúmulo de frutanos se dá sob concentrações mais elevadas de $\mathrm{N}$, nas quais o crescimento é menor. Essa resposta sugere a mobilização de frutanos, que disponibiliza compostos ricos em energia para o crescimento dos órgãos aéreos em $V$. herbacea, como sugerido por Teixeira et al. (1997).

Conforme verificado nos valores de biomassa, o conteúdo de frutanos também variou em função da idade das plantas. Enquanto as plantas tratadas com solução Vernonia com 200\% de força iônica mostraram maior concentração de frutanos aos 90 dias de cultivo, as plantas que receberam soluções mais pobres em nutrientes mostraram aumento no conteúdo de frutanos aos 180 dias. Esses resultados se refletiram na produção de frutanos por planta, tendo sido registrado melhor rendimento em soluções mais pobres em nutrientes no final do experimento (Figura 1), especialmente na solução Vernonia com $50 \%$ de força iônica.

Embora muitas espécies adaptadas a solos oligotróficos respondam ao aumento de nutrientes no solo, elas possuem um limite de tolerância aos níveis mais elevados. Nesse aspecto, a solução de Hoagland $200 \%$ afetou plantas jovens de $V$. herbacea, tendo provocado efeito tóxico, seguido de morte, antes de atingirem dois meses de idade. Esse efeito também foi observado em plantas jovens de Qualea grandiflora por Felippe \& Dale (1990) e de Dalbergia miscolobium (Sassaki \& Felippe, 1998), porém quando cultivadas em solução de Hoagland com 100\% de força iônica, entre 60 e 90 dias de cultivo. Por sua vez, plantas de Bidens gardneri cresceram melhor na solução de Hoagland 200\% (Klein et al., 1996). A causa da mortandade nos casos referidos ainda é desconhecida. Possível efeito tóxico de elementos como o fósforo foi sugerido por Felippe \& Dale (1990), em plantas de Q. grandiflora, uma vez que a concentração de fósforo na solução de Hoagland é de $1 \mathrm{mmol} \mathrm{L}^{-1}$, considerada bem acima da encontrada em solos agrícolas.

Apesar de ser uma espécie herbácea, as respostas de crescimento aos tratamentos nutricionais observadas são comparáveis aos resultados reportados para Q. grandiflora (Felippe \& Dale, 1990) e D. miscolobium (Sassaki \& Felippe, 1998), em que nenhuma diferença foi verificada, até o segundo mês do crescimento inicial, em resposta à adição de nutrientes. No caso de $D$. miscolobium, as diferenças só vieram a ocorrer após o esgotamento das reservas dos cotilédones. Esses resultados sugerem que a idade da planta pode levar a diferentes respostas associadas à disponibilidade de reservas, seja nos cotilédones, seja em outros órgãos de reserva como, por exemplo os rizóforos. Em plantas de cerrado, particularmente, fases mais avançadas de crescimento parecem ser mais adequadas para avaliação do efeito de nutrientes.

Deve-se considerar que, em geral, a razão Ca:Mg é da ordem de 2 a 4 na maioria das soluções nutritivas e em algumas soluções de solo (Parker \& Norvell, 1999). No entanto, semelhante ao solo de cerrado, a solução Vernonia possui Ca:Mg menor que 2, o que indica deficiência de Ca ou toxicidade de Mg (Parker \& Norvell, 1999). Essa característica pode justificar a ampla ocorrência de $V$. herbacea em solos pobres em bases trocáveis, como os de cerrado.

Em relação aos resultados anteriormente obtidos, relacionando o crescimento e a produção de frutanos em V. herbacea (Teixeira et al., 1997; Carvalho et al., 1998; Cuzzuol et al., 2003), este estudo apresenta uma solução nutritiva elaborada especialmente para o cultivo de plantas dessa espécie, com vistas à sua produtividade.

\section{Conclusões}

1. Plantas adaptadas a solos oligotróficos, como Vernonia herbacea, são menos exigentes em nutrientes para o seu crescimento.

2. A solução Vernonia diluída é recomendada para a produção de rizóforos e de frutanos, enquanto a de Hoagland (100\%) e Vernonia (100\%), para crescimento da parte aérea.

\section{Agradecimentos}

À Fapesp, pelo apoio financeiro; ao CNPq, pelas bolsas de pesquisa de Lilian Beatriz Penteado Zaidan e Pedro Roberto Furlani; à Capes, pela concessão de bolsa a Geraldo Rogério Faustini Cuzzuol.

\section{Referências}

CARVALHO, M.A.M.; DIETRICH, S.M.C. Variation in fructan content in the underground organs of Vernonia herbacea (Vell.) Rusby at different phenological phases. New Phytologist, v.123, p.735740, 1993. 
CARVALHO, M.A.M.; PINTO, M.M.; FIGUEIREDO-RIBEIRO, R.C.L. Inulin production by Vernonia herbacea as influenced by mineral fertilization and time of harvest. Revista Brasileira de Botânica, v.21, p.275-280, 1998.

CUZZUOL, G.R.F.; CAMPOS, A. Aspectos nutricionais na vegetação do estuário do Rio Mucuri, Bahia, Brasil. Revista Brasileira de Botânica, v.24, p.227-234, 2001.

CUZZUOL, G.R.F.; CARVALHO, M.A.M.; BARBEDO, C.J.; ZAIDAN, L.B.P. Crescimento e conteúdo de frutanos em plantas de Vernonia herbacea (Vell.) Rusby submetidas à adubação nitrogenada. Revista Brasileira de Botânica, v.26, p.81-91, 2003.

FELIPPE, G.M.; DALE, J.E. The effects of phosphate supply on growth of plants from the Brazilian Cerrado: experiments with seedlings of the annual weed, Bidens gardneri Baker (Compositae) and the tree, Qualea grandiflora (Mart.) (Vochysiaceae). Oecologia, v.82, p.81-86, 1990.

FURLANI, P.R.; SILVEIRA, L.C.P.; BOLONHEZI, D.; FAQUIN, V. Cultivo hidropônico de plantas. Boletim Técnico 180. Instituto Agronômico, Campinas, 1999. 52p.

GOMES, M.A.N.; SHEPHERD, S.L.K. Estudo de nutrição mineral in vitro relacionado à adaptação de Sinningia allagophylla (Martius) Wiehler (Gesneriaceae) às condições de cerrado. Revista Brasileira de Botânica, v.23, p.153-160, 2000.

GRIME, J.P.; HUNT, R. Relative growth-rate: its range and adaptive significance in a local flora. Journal of Ecology, v.63, p.393-422, 1975.

HENDRY, G.A.; WALLACE, R.K. The origin, distribution and evolutionary significance of fructans. In: SUZUKI, M.; CHATTERTON, N.J. (Ed.). Science and technology of fructans. Boca Raton, Tokyo: CRC Press, 1993. p.119-139.

HOAGLAND, D.R.; ARNON, D.I. The water-culture method for growing plants without soil. California Agricultural Experimental Station. Circ. n.347, 1938.

HUNT, R. Plant growth curves: the functional approach to plant growth analysis. London: Edward Arnold Publishers, 1982. 80p.

JERMYN, M.A. A new method for the determination of ketohexoses in presence of aldohexoses. Nature, v.177, p.38-39, 1956.

KLEIN, A.L.; ZAIDAN, L.B.P.; FELIPPE, G.M. Interaction between soil and photoperiod on development of Bidens gardneri Baker (Asteraceae), a herbaceous species from the Brazilian Cerrado. Revista Brasileira de Botânica, v.19, p.1-5, 1996.
LAMBERS, J.; CHAPIN, F.S.; PONS, T.L. Plant physiological ecology. New York: Springer-Verlag, 1998. 540p.

MARSCHNER, H. Mineral nutrition of higher plants. $2^{\text {nd }} e d$. Orlando: Academic Press, 1995. 649p.

MARTINEZ, H.E.P. Formulações de soluções nutritivas para cultivos hidropônicos comerciais. Jaboticabal: Funep, 1997. 31p.

McGRATH, V.B.; BLAKENEY, A.B.; BATTEN, G.D. Fructan to nitrogen ratio as an indicator of nutrient stress in wheat crops. New Phytologist, v.136, p.145-152, 1997.

MORAES, R.M.; DOMINGOS, M. Elementos minerais em folhas de espécies arbóreas de Mata Atlântica e Mata de Restinga, na Ilha do Cardoso, SP. Revista Brasileira de Botânica, v.20, p.133-138, 1997.

PARKER, D.R.; NORVELL, W.A. Advances in solution culture methods for plant mineral nutrition research. In: SPARKS, D.L. (Ed.). Advances in Agronomy. New York: Academic Press, 1999. vol.65, p.151-213.

PAULILO, M.T.S.; FELIPPE, G. Resposta de plântulas de Qualea grandiflora Mart., uma espécie arbórea de cerrado, à adição de nutrientes minerais. Revista Brasileira de Botânica, v.18, p.109112, 1995.

RAIJ, B. van; CANTARELLA, H.; QUAGGIO, J.A.; FURLANI, A.M.C. Recomendações de adubação e calagem para o Estado de São Paulo. Boletim Técnico, 100. 2.ed. Campinas, Instituto Agronômico/ Fundação IAC, 1997. 285p.

SASSAKI, R.M.; FELIPPE, G.M. Response of Dalbergia miscolobium Benth. seedlings, a cerrado tree species, to mineral nutrient supply. Revista Brasileira de Botânica, v.21, p.65-72, 1998.

TEIXEIRA, P.G.; CARVALHO, M.A.M.; ZAIDAN, L.B.P.; KLEIN, A.L. Effect of mineral nutrients on growth and fructan contents in plants of Vernonia herbacea. Revista Brasileira de Fisiologia Vegetal, v.9, p.89-96, 1997.

TERTULIANO, M.F.; FIGUEIREDO-RIBEIRO, R.C. Distribution of fructose polymers in herbaceous species of Asteraceae from the Cerrado. New Phytologist, v.123, p.741-749, 1993.

TOMOMATSU, H. Health effects of oligosaccharides. Food Technology, v.48, p.61-65, 1994.

ZONTA, E.P.; MACHADO A.A.; SILVEIRA JÚNIOR, P. Sistema de análise estatística para microcomputadores (SANEST). Pelotas: UFPel, 1984.

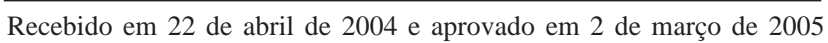

